\title{
Fixed-Node Quantum Monte Carlo Method for Lattice Fermions
}

\author{
H.J.M. van Bemmel, D.F.B. ten Haaf, W. van Saarloos, J.M.J. van Leeuwen, and G. An* \\ Institute Lorentz, Leiden University, P.O. Box 9506, 2300 RA Leiden, The Netherlands
}

(Received 15 December 1993)

\begin{abstract}
We give a new prescription for performing random walks in configuration space for lattice fermion problems. Imposing a suitable condition for the wave function on nodal boundaries in configuration space enables us to devise a generalization of the fixed-node quantum Monte Carlo method, as it has been developed for continuum problems. It does not suffer from the sign problem and provides upper bounds for the energy of different candidates for the ground state. We present new results for the Hubbard model off half filling as a demonstration of the method.

PACS numbers: $71.20 . \mathrm{Ad}, 74.20 . \mathrm{Mn}, 75.10 . \mathrm{Jm}$
\end{abstract}

Interacting electrons are key ingredients for understanding the properties of various classes of materials, ranging from the energetically most favorable shape of small molecules to the magnetic and superconducting instabilities of lattice electron systems, such as high- $T_{\mathrm{c}}$ and heavy fermion compounds. These properties are difficult to extract from even the most simple model Hamiltonians. The reason is that exact diagonalizations are restricted to relatively small systems, while quantum Monte Carlo (QMC) methods are plagued by what is commonly referred to as the sign problem $[1,2]$.

The origin of the sign problem is that the Pauli principle dictates a sign change of the wave function under permutation of any pair of fermions. As a result, when the ground-state energy or the partition function of a fermion model is calculated by means of a QMC method, one obtains contributions of different sign that tend to cancel. Although the problem surfaces in different ways in different methods, there is one common underlying problem. One can keep track of the sign of each contribution, but one needs to determine a difference between the various contributions that is exponentially smaller than the positive and the negative contribution separately [e.g., in the case of a partition function, this ratio is proportional to $\exp (-c \beta N)$, where $\beta=1 / k_{\mathrm{B}} T, N$ the number of fermions, and $c$ a constant]. In practice, this severely limits the applicability of such QMC methods to relatively high temperatures and small system sizes [1,2], except in cases (like the half-filled Hubbard model [3] and the onedimensional Hubbard model [4]) in which the fermion problem can be mapped onto a boson problem.

Ways to deal with the sign problem are under active investigation $[1,2,5-7]$. In the so-called grandcanonical MC method [6], one first performs a HubbardStratonovich transformation, or a discrete equivalence [3], and then integrates out the fermions. This leaves a boson problem. Although a boson system by itself does not have a sign problem, the determinant that comes in via the transformation causes large positive and negative contributions to be present; this partial cancellation tends to make the final answer inaccurate. There is a proposal [7] to take absolute values of the sampled quan- tities, while keeping track of the "average sign." Here, the problem is that the average sign rapidly vanishes for low temperatures. Fahy and Hamann [8] have recently elucidated the intricacies of the sign problem by formulating grand-canonical $\mathrm{MC}$ as a diffusion problem in the space of Slater determinants, and comparing it to Green's function MC (GFMC), which can be formulated as a diffusion problem in configuration space.

In the GFMC method, the ground-state wave function is filtered out from a trial wave function by applying a suitable projection operator to it. This method has been successfully applied to the calculation of ground-state properties of various models, such as the two-dimensional antiferromagnetic Heisenberg model $[1,9]$, but a recent review [1] conjectured that "it is likely that the minussign problem is detrimental for the GFMC method to be applicable to lattice fermion problems."

In this paper we take up this challenge and show that a fixed-node GFMC method can be successfully developed for lattice fermions. The main idea of a fixed-node method $[10,11]$ is to force the random walkers, with which the wave function is sampled, not to cross nodal boundaries, where the wave function changes sign. These nodal boundaries have to be provided by a trial wave function $\psi_{T}$. Since each walker only samples a nodal region where the wave function is of one sign, there is no partial cancellation of contributions with different sign, and good statistics can be obtained. The method gives an estimate for the ground-state energy and wave function (with the proper fermion antisymmetry), given the location of the nodal boundary of $\psi_{T}$. As a result, the method is variational in that it gives a true upper bound to the exact ground-state energy.

The fixed-node GFMC method was developed by Ceperley and Alder [10] for the case in which the electron coordinates are continuous variables, as is the case in the electron gas. It gives very accurate values for the groundstate energies of small molecules [11]. In the continuum approach, the Schrödinger equation is solved exactly in each nodal region, with the boundary condition that the wave function vanishes identically on the nodal boundary of $\psi_{T}$, a surface of codimension 1 in configuration 
space [12]. Extending the method to lattice fermions is far from trivial, since $\psi_{\mathrm{T}}$ generally changes sign in between points of a discrete configuration space. As a result, a lattice Hamiltonian fundamentally connects points in different nodal regions, and to implement the fixednode idea, one is forced to simulate a different effective Hamiltonian $\mathcal{H}_{\text {eff }}$ than the Hamiltonian $\mathcal{H}$ whose groundstate energy one wants to calculate [13]. In the earlier approach by two of us [13], this made the method nonvariational, but we show here that it is possible to introduce a "nodal boundary potential term" $V_{\text {nb }}$ in $\mathcal{H}_{\text {eff }}$ which makes the method variational.

We now outline our method. In GFMC, one applies a projection operator $\mathcal{F}=1-\tau(\mathcal{H}-w)$ to filter out the ground state from a trial wave function. After $n$ iterations the trial state has evolved to

$$
\left|\psi^{n}\right\rangle=\mathcal{F}^{n}\left|\psi_{T}\right\rangle=[1-\tau(\mathcal{H}-w)]^{n}\left|\psi_{T}\right\rangle .
$$

Choosing $w$ close to the ground-state energy [14] and taking $\tau$ small enough [15] ensures that eventually the ground state will dominate. This projection is implemented in configuration space $\{R\}$, which should not be confused with the original lattice in real space: a point in $\{R\}$ specifies the positions of all the labeled fermions on the real lattice. For the wave function at point $R$ we write $\psi^{n}(R)=\left\langle R \mid \psi^{n}\right\rangle$, and for the Hamiltonian operator $H\left(R, R^{\prime}\right)=\left\langle R|\mathcal{H}| R^{\prime}\right\rangle$. To obtain good statistics, importance sampling is used. The Green's function

$$
G\left(R, R^{\prime}\right)=\psi_{T}(R) F\left(R, R^{\prime}\right) \psi_{T}^{-1}\left(R^{\prime}\right)
$$

is introduced. The mixed estimate for the expectation value of an operator $\mathcal{O}$ then becomes $\left\langle\psi^{n}|\mathcal{O}| \psi_{T}\right\rangle$ $\times\left\langle\psi^{n} \mid \psi_{T}\right\rangle^{-1}$, where [16]

$$
\left\langle\psi^{n}|\mathcal{O}| \psi_{T}\right\rangle=\sum_{\mathcal{R}} O\left(R_{n}\right) \prod_{i=1}^{n} G\left(R_{i}, R_{i-1}\right) \psi_{T}^{2}\left(R_{0}\right) .
$$

Here $O(R) \equiv\left\langle R|\mathcal{O}| \psi_{T}\right\rangle \psi_{T}^{-1}(R)$ is the local value of $\mathcal{O}$, and $\mathcal{R}=\left\{R_{0}, R_{1}, R_{2}, \ldots, R_{n}\right\}$ is a path in configuration space. In this way, one samples more in regions with large contributions. If we split the Green's function in a part $P$ which satisfies $\sum_{R} P\left(R, R^{\prime}\right)=1$ and a remaining part $m$, which we can choose to depend only on $R^{\prime}$,

$$
G\left(R, R^{\prime}\right)=P\left(R, R^{\prime}\right) m\left(R^{\prime}\right)
$$

we have a random walk interpretation. The stochastic matrix $P$ defines the transition probabilities, and $m$ is the "multiplicity" of the walkers. As discussed by Trivedi and Ceperley [9] and Hetherington [17], in order to obtain a proper statistics the ensemble of walkers is updated regularly by letting walkers die or multiply according to their value of $m . H\left(R, R^{\prime}\right.$ ) (and hence $P$ ) connects points in configuration space. For example, for the Hubbard model with nearest neighbor hopping amplitude $t, H\left(R, R^{\prime}\right)=-t$ if $R^{\prime}$ and $R$ differ by moving

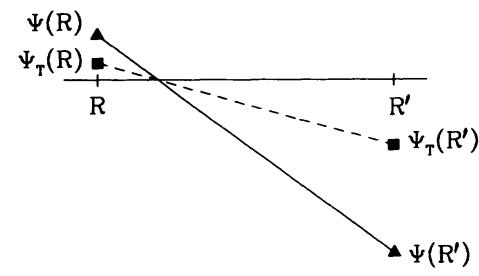

FIG. 1. The "lever rule" of Eq. (5). The point where the linear interpolation of the wave function $\psi$ vanishes must coincide with the point where the linear interpolation of the trial wave function $\psi_{T}$ vanishes.

over one labeled electron by one lattice unit in real space and $H\left(R, R^{\prime}\right)=U N_{D}$ if $R^{\prime}=R$, with $N_{D}$ the number of doubly occupied sites.

If $\psi_{T}$ has different signs in $R$ and in $R^{\prime}$, then $G\left(R, R^{\prime}\right)$ is negative [see Eq. (2)]. This is the cause of the sign problem: an expectation value as calculated in Eq. (3) is a sum of a large number of positive and negative contributions. Therefore, we do not want to perform steps across a nodal boundary. Unlike in the continuum case, where very small steps in time and in configuration space can be made, these steps are fundamentally present in the Hamiltonian of a lattice problem, and therefore in the projection operator $\mathcal{F}$ in Eq. (1).

Our crucial new point is that we make a suitable choice of boundary conditions in configuration space [18]. We require that the wave function to which the system evolves satisfies

$$
\frac{\psi\left(R^{\prime}\right)}{\psi(R)}=\frac{\psi_{T}\left(R^{\prime}\right)}{\psi_{T}(R)}
$$

where $R$ and $R^{\prime}$ are configurations on either side of a node, and where $\psi_{T}$ is the trial wave function. This can be viewed as pinning down the nodal boundary in a point between lattice points. The precise nodal point is the point where a linear interpolation of the trial wave function vanishes. The requirement (5) can be viewed as a "lever rule" for the wave function. This is depicted in Fig. 1.

Now, consider the Schrödinger equation

$$
\sum_{R^{\prime}} H\left(R, R^{\prime}\right) \psi\left(R^{\prime}\right)=E_{0} \psi(R)
$$

The summation over $R^{\prime}$ involves terms with $R^{\prime}$ in the same nodal region as $R$ [we denote this by $R^{\prime} \in \mathcal{N}(R)$ ] and terms with $R^{\prime}$, which can be reached from $R$ by one step allowed by $H\left(R, R^{\prime}\right)$, across the nodal boundary [denoted as $R^{\prime} \in \partial \mathcal{N}(R)$ ]. If we now impose condition (5) and eliminate $\psi\left(R^{\prime}\right)$ in the latter terms, we can write $\mathcal{H} \psi$ instead as $\mathcal{H}_{\text {eff }} \psi$, with

$$
H_{\text {eff }}\left(R, R^{\prime}\right)=\left\{\begin{array}{l}
H\left(R, R^{\prime}\right)+V_{\mathrm{nb}}(R) \delta_{R R^{\prime}}, \quad R^{\prime} \in \mathcal{N}(R), \\
0, \text { otherwise }
\end{array}\right.
$$

where the nodal boundary potential is given by 


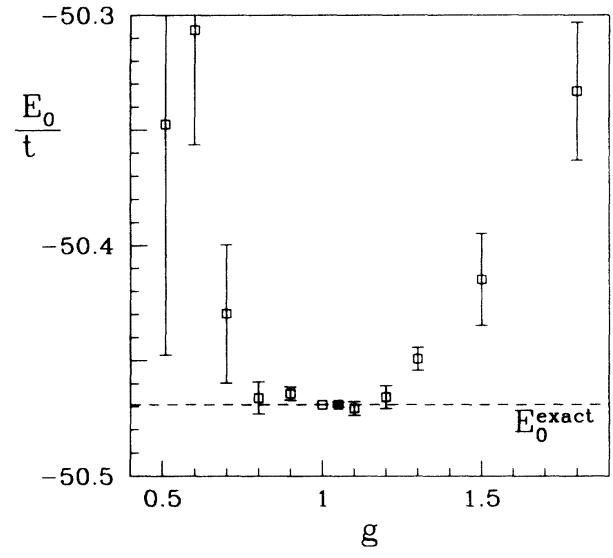

FIG. 2. Fixed-node ground-state energy for a system of free electrons as a function of the Gutzwiller factor that is included in the trial wave function. The error bars give 1 standard deviation in the statistical accuracy. This system has 24 electrons on 32 sites of a square lattice of the type described in Ref. [13].

$$
V_{\mathrm{nb}}(R)=\sum_{R^{\prime} \in \partial \mathcal{N}(R)} H\left(R, R^{\prime}\right) \frac{\psi_{T}\left(R^{\prime}\right)}{\psi_{T}(R)} .
$$

To understand the effect of this term, note that for the Hubbard model the matrix elements $H\left(R, R^{\prime}\right)$ in Eq. (8) equal $-t$, while the ratios $\psi_{T}\left(R^{\prime}\right) / \psi_{T}(R)$ in this sum are negative. Hence $V_{\text {nb }}(R)$ acts as an on-site repulsive potential in configuration space that suppresses the wave function in points which are close to nodes. The same suppression is required by those hops which are present in $\mathcal{H}$ but which are replaced by $V_{\text {nb }}$ in $\mathcal{H}_{\text {eff. }}$. These kinetic terms force the wave function to be smooth and thus to be small near a node. In the continuum limit, the effect of $V_{\mathrm{nb}}$ is equivalent to the usual prescription [10] that $\psi$ vanishes on the nodal boundary.

The terms in the mixed estimator $\left\langle\psi_{0}|\mathcal{H}| \psi_{T}\right\rangle$, associated with hops across the nodal surface, are identical to those arising from the nodal potential in $\left\langle\psi_{0}\left|\mathcal{H}_{\text {eff }}\right| \psi_{T}\right\rangle$; hence the ground-state energy expectation values of the Hamiltonians are the same: $\left\langle\psi_{0}|\mathcal{H}| \psi_{T}\right\rangle=\left\langle\psi_{0}\left|\mathcal{H}_{\text {eff }}\right| \psi_{T}\right\rangle$. Therefore, we can do the simulation entirely with $\mathcal{H}_{\text {eff }}$, which only contains hops within one nodal region. Solving for the ground state of $\mathcal{H}_{\text {eff }}$ and measuring the energy of the same Hamiltonian in that state, one therefore does not encounter a sign problem, even though by construction the wave function so obtained does have the proper fermion antisymmetry. This procedure gives the lowest energy of the true Hamiltonian in a restricted space of wave functions with a specified nodal structure. This implies that we have a variational principle (cf. Ref. [11], section D).

It is instructive to first illustrate our approach by considering free electrons, that is, the Hubbard model with $U=0$. The exact wave function is a Slater determi-

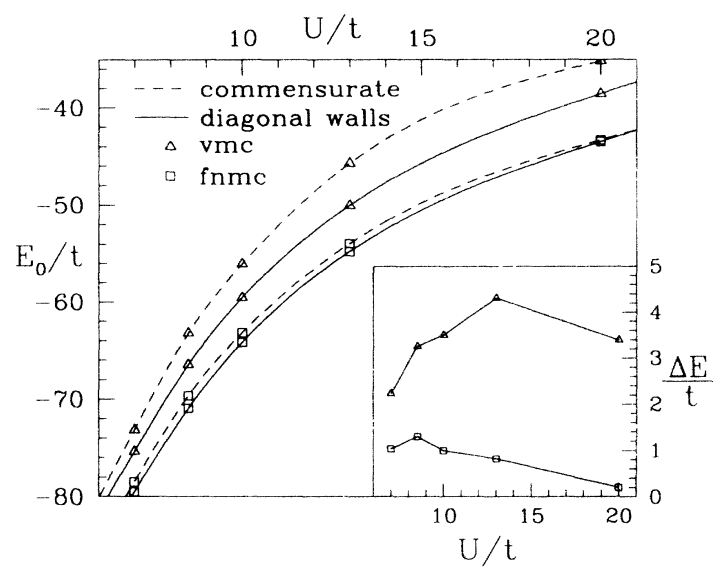

FIG. 3. Ground-state energies for a system of 104 electrons on a 112-site lattice. To study diagonal domain-wall states, a lattice with periodic boundary conditions in the diagonal directions is used, as described in Ref. [13]. Solid lines, diagonal-wall state; dashes lines, commensurate state. Triangles, variational Monte Carlo; squares, fixed-node Monte Carlo. The lines are a guide for the eye, obtained by a spline through the data points. The statistical errors are smaller than the symbol size (1 standard deviation of the energy is smaller than 0.1). The inset shows the energy difference $\Delta E=E_{0}^{\mathrm{cm}}-E_{0}^{\mathrm{dw}}$, for variational (triangles) and fixed-node (squares) Monte Carlo. Here, the statistical error can be estimated from the scattering of the data points.

nant of single-particle wave functions, but instead we take an approximate trial wave function by including a Gutzwiller factor [19]. When the nodal boundary lies between two points in configuration space with a different number of doubly occupied sites, the nodal boundary is shifted away from its exact location when $g \neq 1$. The results in Fig. 2 illustrate the variational character of our method: the energy is above the exact value for all $g \neq 1$. Nevertheless, the minimum is relatively flat, indicating some insensitivity to the exact location of the nodal boundary, and the statistical errors for $g$ not too different from 1 are small.

We have used this method to investigate the stability of diagonal domain walls in the ground state of the two-dimensional Hubbard model. Hartree-Fock [20] and variational Monte Carlo [21] calculations indicate that such structures on a mesoscopic scale are present. Our question is whether this inhomogeneous ground state is still stable with respect to a homogeneous one, if both possible phases are allowed to lower their energy because much more fluctuations (only restricted by the nodes of the trial wave function) are taken into account in the simulations. Taking the variational Monte Carlo results [21] for the trial wave functions, we have calculated the energies as a function of $U$ at doping $\delta=0.07$. The results are presented in Fig. 3.

The upper bounds for the energy are lower than those of variational Monte Carlo and Hartree-Fock calcula- 
tions. One also concludes that the stability of domain walls is diminished compared to these previous calculations. The reason is that Hartree-Fock and variational Monte Carlo calculations tend to overestimate the influence of potential energy effects, compared to kinetic energy effects. The presence of domain walls corresponds to a gain in magnetic energy compared to a homogeneous phase and to a loss in kinetic energy. Allowing for more fluctuations than in previous calculations reveals that the presence of walls is less favorable than these earlier calculations indicate. The ground state with walls is still stable, however. Note that our energies are within the statistical accuracy of the results of Ref. [13], while our present method is variational and in addition allows us to check self-consistently that $w$ in Eq. (1) coincides with $E_{0}$.

In conclusion, we have shown that the continuum fixednode GFMC can be successfully extended to the calculation of ground-state properties of lattice fermions. Clear merits of the method are that it avoids the sign problem and that it obeys a variational principle. Other advantages are that it does not rely on a model-specific transformation, and that it allows one to systematically improve on existing approximate theories (e.g., HartreeFock).

Besides performing further tests of our approach, e.g., by comparing with exact results, other important issues for future research are the development of criteria indicating which trial wave function constitutes a good approximation for the structure of the nodal regions, and the question whether a form of "nodal release" [10] can be developed.

We acknowledge fruitful discussions with P.J.H. Denteneer. Part of this research was supported by the Stichting voor Fundamenteel Onderzoek der Materie (FOM), which is financially supported by the Nederlandse Organisatie voor Wetenschappelijk Onderzoek (NWO).

* Present address: Shell Research, P.O. Box 60, 2280 AB, Rijswijk, The Netherlands.

[1] H. De Raedt and W. von der Linden, in The Monte Carlo Method In Condensed Matter Physics, edited by
K. Binder (Springer-Verlag, Berlin, 1992).

[2] M. Suzuki, Physica (Amsterdam) 194A, 432 (1993).

[3] J.E. Hirsch, Phys. Rev. B 31, 4403 (1985).

[4] M.A. Lee, K.A. Motakabbir, and K.E. Schmidt, Phys. Rev. Lett. 53, 1191 (1984).

[5] M. Boninsegni and E. Manousakis, Phys. Rev. B 46, 560 (1992).

[6] E.Y. Loh, Jr., J.E. Gubernatis, R.T. Scalettar, S.R. White, D.J. Scalapino, and R.L. Sugar, Phys. Rev. B 41, 9301 (1990).

[7] S. Sorella, S. Baroni, R. Car, and M. Parrinello, Europhys. Lett. 8, 663 (1989).

[8] S. Fahy and D.R. Hamann, Phys. Rev. B 43, 765 (1991).

[9] N. Trivedi and D.M. Ceperley, Phys. Rev. B 41, 4552 (1990).

[10] D.M. Ceperley and B.J. Alder, Phys. Rev. Lett. 45, 566 (1980); Science 231, 555 (1986).

[11] P.J. Reynolds, D.M. Ceperley, B.J. Alder, and W.A. Lester, Jr., J. Chem. Phys. 77, 5593 (1982).

[12] When the Hamiltonian is not time-reversal invariant, as is the case in the presence of a magnetic field, the continuum fixed-node GFMC can be extended to a fixed-phase method. See G. Ortiz, D.M. Ceperley, and R.M. Martin, Phys. Rev. Lett. 71, 2777 (1993).

[13] G. An and J.M.J. van Leeuwen, Phys. Rev. B 44, 9410 (1991).

[14] This is done in practice by adjusting $w$ self-consistently so that it becomes equal to the measured ground-state energy.

[15] $\tau$ should be such that $-1<1-\tau\left(E_{\max }-w\right)$, where $E_{\max }$ is the largest eigenvalue of $\mathcal{H}$. See, e.g., Ref. [13].

[16] While mixed estimators of the form (3) arise most naturally in GFMC approaches, they are only exact if $\mathcal{O}$ commutes with $\mathcal{H}$. See, e.g., Ref. [11].

[17] J.H. Hetherington, Phys. Rev. A 30, 2713 (1984).

[18] A somewhat similar idea has been advanced for continuum problems with a nonlocal potential by L. Mitas, E.L. Shirley, and D.M. Ceperley, J. Chem. Phys. 95, 3467 (1991).

[19] M.C. Gutzwiller, Phys. Rev. Lett. 10, 159 (1963); Phys. Rev. 137, A1726 (1965).

[20] D. Poilblanc and T.M. Rice, Phys. Rev. B 39, 9749 (1989); H.J. Schulz, J. Phys. (Paris) 50, 2833 (1989); K. Machida, Physica (Amsterdam) 158C, 192 (1989); J. Zaanen and O. Gunnarsson, Phys. Rev. B 40, 7391 (1989).

[21] T. Giamarchi and C. Lhuillier, Phys. Rev. B 42, 10641 (1990). 